\title{
Durable response to afatinib in an advanced lung adenocarcinoma patient with an EGFR L858R/G729A compound mutation: a case report
}

\author{
Lige $\mathrm{Wu}^{1 \#}$, Cheng Fang ${ }^{1 \#}$, Weiqing $\mathrm{Zhao}^{1} \wedge$, Dong $\mathrm{Li}^{1}$, Shuxian Tang ${ }^{1}, \mathrm{Xi}^{2}{ }^{2} \wedge$, Mei Ji ${ }^{1} \wedge$ \\ ${ }^{1}$ Department of Oncology, Third Affiliated Hospital of Soochow University, Changzhou, China; ${ }^{2}$ Burning Rock Biotech, Guangzhou, China \\ \#These authors contributed equally to this work. \\ Correspondence to: Mei Ji, MD. Department of Oncology, Third Affiliated Hospital of Soochow University, 185 Juqian Street, Changzhou 213003 , \\ China. Email: jimei_90@126.com.
}

\begin{abstract}
Of the epidermal growth factor receptor $(E G F R)$ mutations in patients with non-small cell lung cancer (NSCLC), 10-15\% are uncommon mutations. Most of the EGFR "major" uncommon mutations have shown responses to EGFR-tyrosine kinase inhibitors (TKIs). However, there is a lack of clinical data for other less common types of EGFR mutations and the response to EGFR-TKIs, occurring either alone or in combination with EGFR sensitizing mutations. We reported a 70-year-old Chinese man with no smoking history who was diagnosed with stage IVA lung adenocarcinoma. An exceptionally uncommon EGFR G729A mutation in EGFR exon 19 was detected concomitantly with EGFR L858R in exon 21 in tumor specimens by next generation sequencing (NGS). This patient obtained limited benefit from icotinib and the increase in symptoms of cough and chest tightness, so we decided to switch the treatment to afatinib. Our patient exhibited partial response to afatinib with progression-free survival of 10 months. Subsequently, an EGFR T790M mutation was detected in the second lung biopsy. Then, osimertinib was administered and the symptoms improved significantly and the progress-free survival was nearly 16 months. Our data suggests that patients with NSCLC who are positive for uncommon EGFR G729A mutations may benefit from treatment with afatinib.
\end{abstract}

Keywords: Non-small cell lung cancer (NSCLC); uncommon mutation; afatinib; EGFR G729A; case report

Submitted Nov 09, 2021. Accepted for publication Jan 13, 2022.

doi: $10.21037 /$ atm-22-95

View this article at: https://dx.doi.org/10.21037/atm-22-95

\section{Introduction}

Genetic alterations in epidermal growth factor receptor (EGFR) constitute a major oncogenic mechanism in non-small cell lung cancer (NSCLC) (1). The frequency of EGFRpositive mutations is approximately $10 \%$ to $15 \%$ among Caucasians and 30\% to 50\% among Asians with NSCLC (2). Patients with common EGFR-mutant NSCLC, including deletions in exon 19 (19del) and Leu858Arg (L858R), account for $85-90 \%$ of the EGFR mutations (3). The remaining
10-15\% EGFR mutations are composed of a heterogeneous group of "major" rare mutations (i.e., G719X, S768I, L861Q), very rare mutations (major rare mutations negative), and compound gene alterations within exons 18-21 (4). Given the significant heterogeneity among uncommon EGFR mutations, their responses to EGFR-tyrosine kinase inhibitors (TKIs) are also different. Thus, individualized treatment for each case is strongly recommended. Afatinib is the second-generation EGFR-TKI, which block ErbB family receptors irreversible. Previous study indicated that afatinib

\footnotetext{
^ ORCID: Lige Wu, 0000-0002-6785-2430; Cheng Fang, 0000-0003-0974-3516; Weiqing Zhao, 0000-0002-6066-3311; Xi Li, 0000-00026391-901X; Mei Ji, 0000-0001-8137-1441.
} 

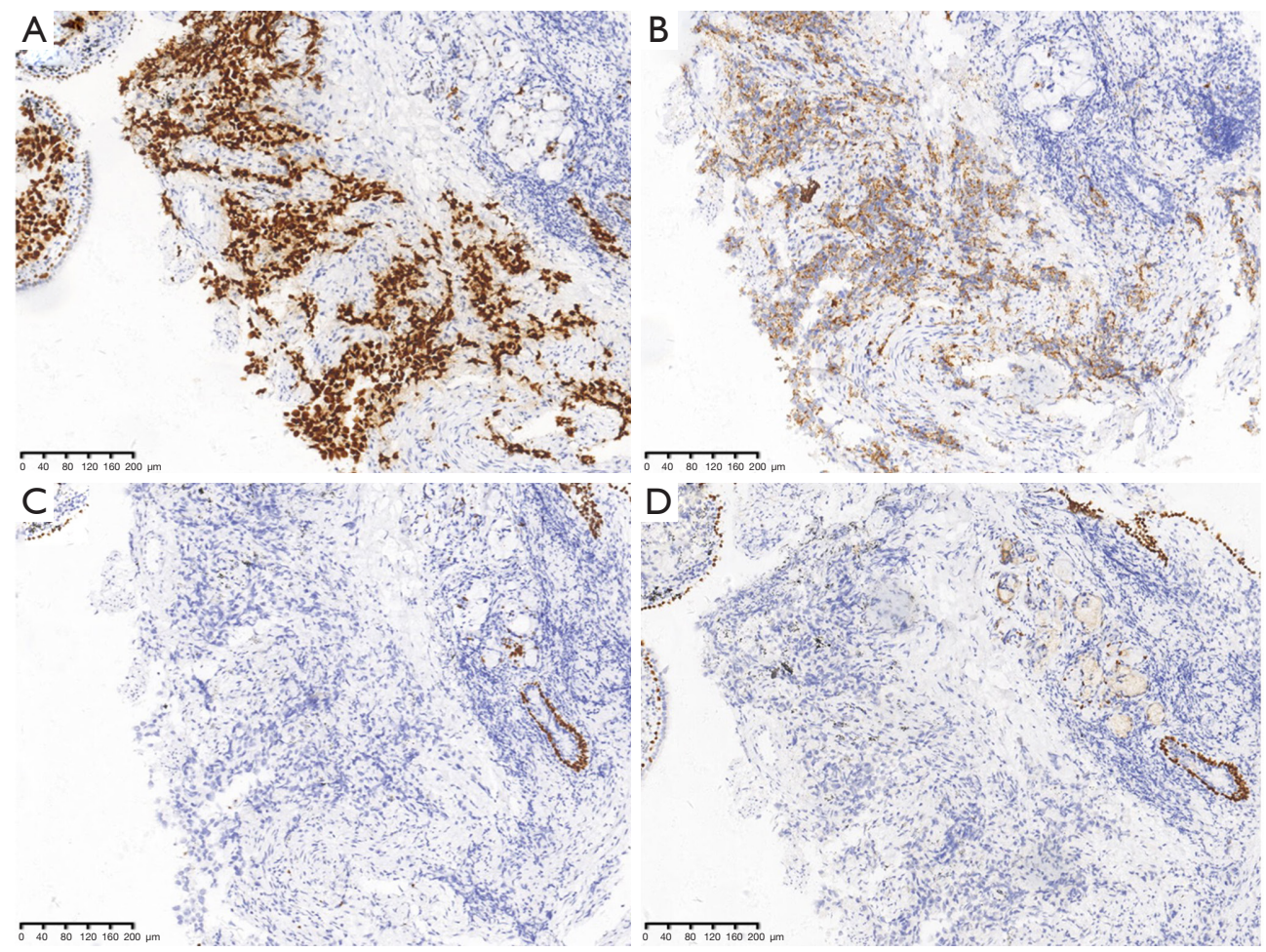

Figure 1 Pathological examination of lung biopsies revealed metastatic lung adenocarcinoma. Immunostaining of TTF1 (A), Napsin A (B), P40 (C) and P63 (D) in tissues.

combined with bevacizumab had good effects on NSCLC patient with brain metastasis and EGFR del 18 mutation; but the effect of bevacizumab cannot be eliminated (5). In this study, we reported a case of a patient with advanced stage NSCLC harboring a rare EGFR L858R/G729A compound mutation, in which the second-generation EGFR-TKI afatinib monotherapy elicited more durable responses than the initial icotinib treatment. We present the following case in accordance with the CARE reporting checklist (available at https://atm.amegroups.com/article/view/10.21037/atm$22-95 / \mathrm{rc})$.

\section{Case presentation}

A 70-year-old Chinese man with no smoking history presented to our hospital with 1 month of intermittent coughing. A contrast-enhanced computed tomography (CT) scan revealed a $5 \mathrm{~cm} \times 4.1 \mathrm{~cm}$ mass located in the left upper lung lobe. A bone scan showed ilium metastasis. Immunohistochemistry (IHC) indicated that most of the tumor cells were positive for thyroid transcription factor-1 (TTF-1) and Napsin A (Figure 1). After comprehensive evaluation, the patient was diagnosed with stage IVA (cT2bN0M1b) lung adenocarcinoma with bone metastasis. Capture-based targeted sequencing performed on formalinfixed paraffin-embedded (FFPE) samples of lung lesions indicated the presence of complex mutations: EGFR L858R in exon 21 and G729A in exon 19, with mutation frequencies of $16.77 \%$ and $12.48 \%$, respectively (Figure 2).

The patient was administered with icotinib as a firstline treatment at $125 \mathrm{mg}$ orally TID from Nov 2018. From a CT scan in Mar 2019, the patient was assessed as stable disease (SD) based on the Response Evaluation Criteria on Solid Tumors version (RECIST) version 1.1 (Figure 3). Because of inadequate treatment response, the dosage of icotinib was increased to $250 \mathrm{mg}$ orally TID from Jun 2019. After another 3 months of treatment, the patient developed severe clinical symptoms including worsening cough and shortness of breath. Follow-up CT scans revealed slight enlargement of the primary lesion $(5.4 \mathrm{~cm} \times 4.9 \mathrm{~cm})$ and mediastinal lymph nodes compared with previous images (Figure $3 A, 3 B$ ). Although an assessment of progressive disease $(\mathrm{PD})$ could not be made based on the CT scans, the physician decided to switch the treatment to afatinib 

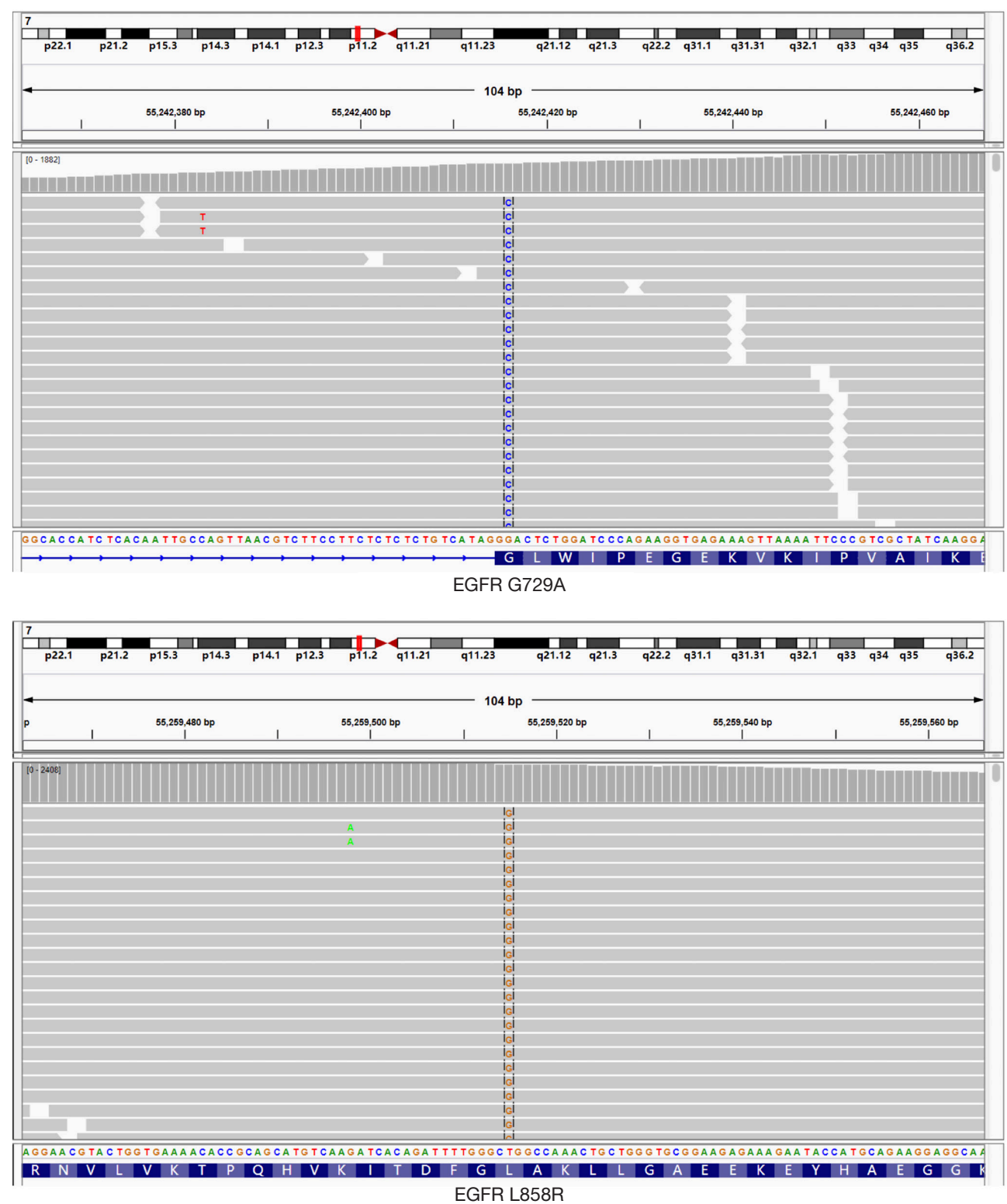

Figure 2 The IGV images representing the EGFR compound mutation consisting of L858R and G729A in the lung lesion samples. IGV, integrative genomics viewer; EGFR, epidermal growth factor receptor.

due to the limited benefit from icotinib and the increase in symptoms of cough and chest tightness. The total treatment duration of icotinib was 10 months.

Subsequently, the patient was placed on afatinib at $40 \mathrm{mg}$ once daily from Sep 2019. Four weeks after the initiation of afatinib, CT scans revealed remarkable tumor shrinkage of the left lung mass $(3 \mathrm{~cm} \times 3 \mathrm{~cm}$; Figure $3 C)$. The patient achieved partial response (PR). CT scans 5 months later showed further shrinkage in the primary pulmonary lesion
$(2.8 \mathrm{~cm} \times 2.6 \mathrm{~cm}$; Figure $3 D)$. There was no serious adverse effect during the course of afatinib therapy. The patient's clinical symptoms were also alleviated.

After 10 months of afatinib treatment in total, chest $C T$ scans revealed a marked increase in tumor size that led to PD $(5.2 \mathrm{~cm} \times 3.7 \mathrm{~cm})$. EGFR T790M mutation was detected in the second lung biopsy. The progression-free survival (PFS) of afatinib was 10 months. The treatment was switched to osimertinib at $80 \mathrm{mg}$ once daily from Jul 2020, 

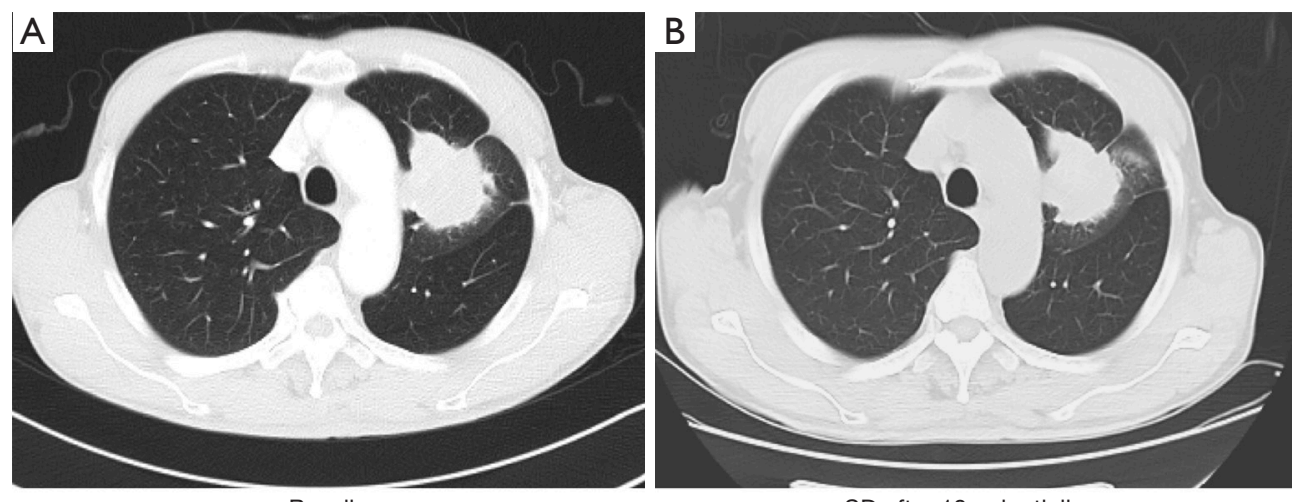

Baseline

SD after $10 \mathrm{~m}$ icotinib

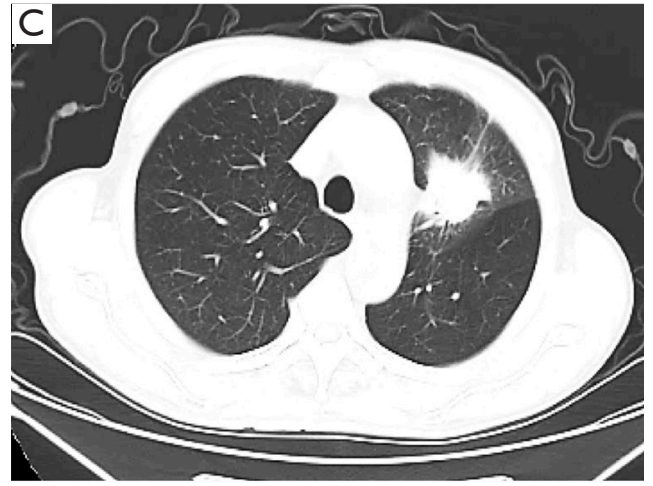

PR after $1 \mathrm{~m}$ of afatinib

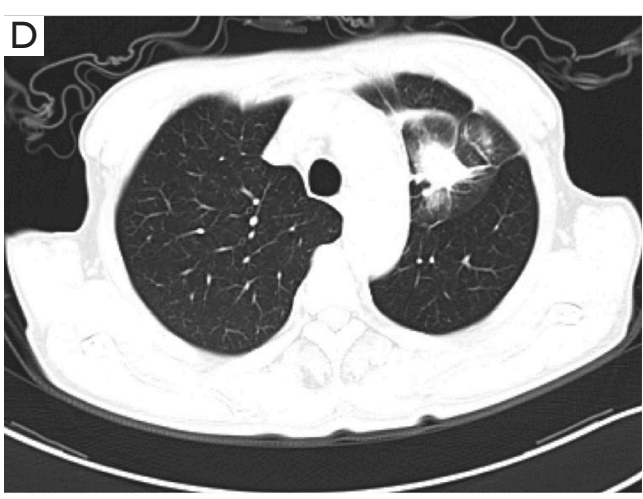

PR after $6 \mathrm{~m}$ of afatinib

Figure 3 The CT images of the primary lung mass at baseline (A), at the evaluation of SD after 10 months of icotinib treatment (B), at the evaluation of PR after 1 month of afatinib treatment (C), and at the evaluation of PR after 6 months of afatinib treatment (D). SD, stable disease; PR, partial response.

with the patient's disease remaining stable for 16 months as of his last follow-up in October 2021. Timeline of the patient's treatment history was presented in Figure 4.

All procedures performed in this study were in accordance with the ethical standards of the institutional and/or national research committee(s) and with the Helsinki Declaration (as revised in 2013). Written informed consent was obtained from the patient for publication of this manuscript and any accompanying images. A copy of the written consent is available for review by the editorial office of this journal.

\section{Discussion}

This case reported the enhanced clinical efficacy of afatinib compared to icotinib in a patient with metastatic NSCLC carrying an EGFR L858R/G729A compound mutation. Although the patient achieved SD and never progressed radiologically during the course of icotinib treatment, his primary tumor was enlarging slowly with the exacerbation of clinical symptoms. After switching to afatinib, the patient achieved PR as evidenced by the alleviation of clinical symptoms as well as shrinkage of the primary lung lesion. This patient harboring a rare EGFR L858R/ G729A compound mutation did not achieve an objective response to icotinib but appeared to be sensitive to afatinib, suggesting that G729A may contribute to the lack of response to first-generation EGFR-TKIs.

The uncommon G729A mutation has rarely been reported in NSCLC. Only one patient with quadruple mutations of L861Q, L858R, E745_A750del, and G729A has been reported (6). Like the EGFR G719X (where X represents $\mathrm{A}, \mathrm{S}, \mathrm{C}$, or D) mutation, the Gly729 mutation site is also predicted to be part of the phosphate-binding "P-loop" of the EGFR tyrosine kinase domain, which contributes to the hydrophobic cluster surrounding L858 in the inactive state (7). Substitution of the Gly729 amino acid residue with alanine may potentially "tweak" the P-loop and 


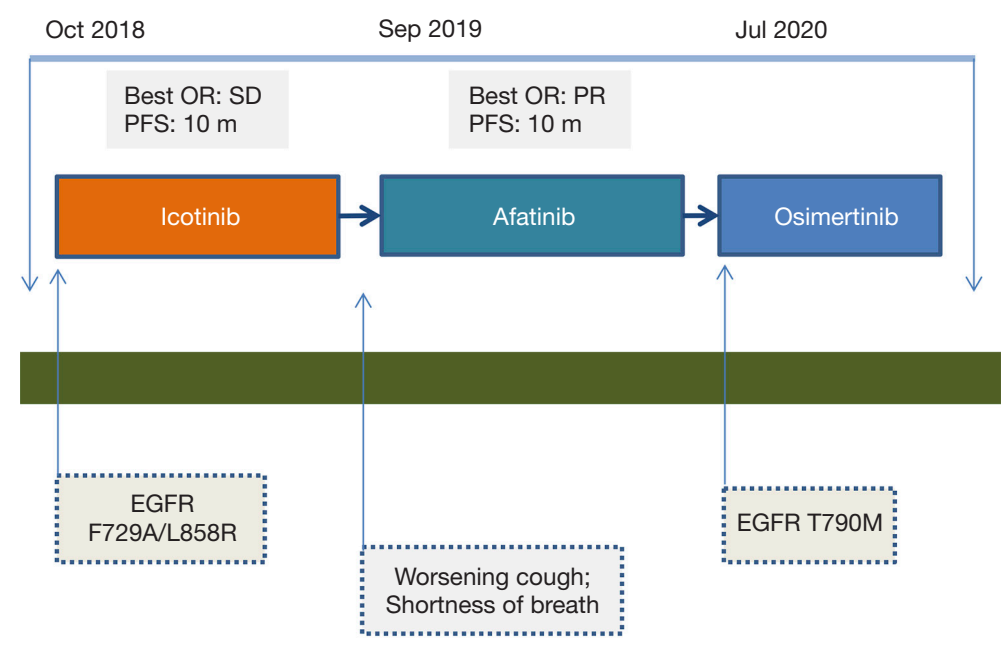

Figure 4 Timeline of the patient's treatment history. OR, best overall response; PFS, progression-free survival; SD, stable disease; PR, partial response; EGFR, epidermal growth factor receptor.

positively regulate the ATP binding affinity to EGFR-TKIs, which suggests that the G729A mutation might be a TKI sensitizing mutation. Although there is limited published research regarding the point mutations in the P-loop, most of the mutations are reported to be sensitive to TKIs including S720P/F, P699S, N700D, E709Q, G721A, and L718P (7). However, one study reported that none of the tumors with the EGFR E709A/G mutation associated with L858R responded to EGFR-TKIs (8). EGFR uncommon mutations showed divergent sensitivities to EGFR-TKIs, making it difficult to predict suitable TKIs for patients harboring uncommon EGFR mutations. This highlights the importance of assessing uncommon EGFR mutations not as a whole group but independently.

Afatinib has generally been shown to exhibit broad activity across EGFR rare mutations (9). Study showed that afatinib was active in patients with certain types of uncommon EGFR mutations, especially G719X, but less active in other uncommon EGFR mutation types because of significant heterogeneity among EGFR mutations (10). An in vitro study showed that $B a / F 3$ cells expressing the G719X EGFR mutation were sensitive to irreversible inhibitors, especially the second-generation EGFR-TKI afatinib, but resistant to first-generation reversible EGFRTKIs (8). In our study, we demonstrated that a patient with metastatic NSCLC harboring a L858R/G729A compound mutation showed a more favorable response to afatinib than to icotinib. This provides clinical evidence supporting that patients with NSCLC carrying "less" uncommon
EGFR mutations may derive a greater benefit from afatinib. Further studies are warranted to examine the efficacy of different EGFR-TKIs in various rare $E G F R$ mutations occurring alone or concomitantly with $E G F R$ sensitizing mutations.

In conclusion, we showed the significant clinical efficacy of the second-generation EGFR-TKI afatinib in a patient with advanced stage NSCLC harboring an exceptionally rare compound EGFR L858R/G729A mutation. This is the first report of a durable response to afatinib in a patient with NSCLC harboring the EGFR G729A mutation. Our data suggests that patients with NSCLC who are positive for uncommon $E G F R$ mutations may benefit from treatment with afatinib. However, as a high degree of molecular heterogeneity is present in the uncommon EGFR mutation population, individualized treatment should be recommended based on existing data for each case in clinical practice.

\section{Acknowledgments}

Funding: This study was supported by the Science and Technology Project for Youth Talent of Changzhou Health Commission (QN201703, QN201722), Young Talent Development Plan of Changzhou Health Commission (CZQM2020024), Major Science and Technology Project of Changzhou Health Commission (ZD202004, ZD202007), and China Postdoctoral Science Foundation (2020M670064ZX). 


\section{Footnote}

Reporting Checklist: The authors have completed the CARE reporting checklist. Available at https://atm.amegroups. com/article/view/10.21037/atm-22-95/rc

Conflicts of Interest: All authors have completed the ICMJE uniform disclosure form (available at https://atm. amegroups.com/article/view/10.21037/atm-22-95/coif). XL is from Burning Rock Biotech. The other authors have no conflicts of interest to declare.

Ethical Statement: The authors are accountable for all aspects of the work in ensuring that questions related to the accuracy or integrity of any part of the work are appropriately investigated and resolved. All procedures performed in this study were in accordance with the ethical standards of the institutional and/or national research committee(s) and with the Helsinki Declaration (as revised in 2013). Written informed consent was obtained from the patient for publication of this manuscript and any accompanying images. A copy of the written consent is available for review by the editorial office of this journal.

Open Access Statement: This is an Open Access article distributed in accordance with the Creative Commons Attribution-NonCommercial-NoDerivs 4.0 International License (CC BY-NC-ND 4.0), which permits the noncommercial replication and distribution of the article with the strict proviso that no changes or edits are made and the original work is properly cited (including links to both the formal publication through the relevant DOI and the license). See: https://creativecommons.org/licenses/by-nc-nd/4.0/.

\section{References}

1. Yatabe Y, Kerr KM, Utomo A, et al. EGFR mutation testing practices within the Asia Pacific region: results of a multicenter diagnostic survey. J Thorac Oncol 2015;10:438-45.

2. Passaro A, Mok T, Peters S, et al. Recent Advances

Cite this article as: Wu L, Fang C, Zhao W, Li D, Tang S, Li X, Ji M. Durable response to afatinib in an advanced lung adenocarcinoma patient with an EGFR L858R/ G729A compound mutation: a case report. Ann Transl Med 2022;10(2):116. doi: 10.21037/atm-22-95 on the Role of EGFR Tyrosine Kinase Inhibitors in the Management of NSCLC With Uncommon, Non Exon 20 Insertions, EGFR Mutations. J Thorac Oncol 2021;16:764-73.

3. Li WQ, Cui JW. Non-small cell lung cancer patients with ex19del or exon 21 L858R mutation: distinct mechanisms, different efficacies to treatments. J Cancer Res Clin Oncol 2020;146:2329-38.

4. Graham RP, Treece AL, Lindeman NI, et al. Worldwide Frequency of Commonly Detected EGFR Mutations. Arch Pathol Lab Med 2018;142:163-7.

5. An N, Wang H, Zhu H, et al. Great efficacy of afatinib on a patient with lung adenocarcinoma harboring uncommon EGFR delE709_T710insD mutations: a case report. Onco Targets Ther 2019;12:7399-404.

6. Jing C, Mao X, Wang Z, et al. Next-generation sequencing-based detection of EGFR, KRAS, BRAF, NRAS, PIK3CA, Her-2 and TP53 mutations in patients with non-small cell lung cancer. Mol Med Rep 2018;18:2191-7.

7. Massarelli E, Johnson FM, Erickson HS, et al. Uncommon epidermal growth factor receptor mutations in non-small cell lung cancer and their mechanisms of EGFR tyrosine kinase inhibitors sensitivity and resistance. Lung Cancer 2013;80:235-41.

8. Kohsaka S, Nagano M, Ueno T, et al. A method of highthroughput functional evaluation of EGFR gene variants of unknown significance in cancer. Sci Transl Med 2017;9:eaan6566.

9. Yang JC, Schuler M, Popat S, et al. Afatinib for the Treatment of NSCLC Harboring Uncommon EGFR Mutations: A Database of 693 Cases. J Thorac Oncol 2020;15:803-15.

10. Yang JC, Sequist LV, Geater SL, et al. Clinical activity of afatinib in patients with advanced non-small-cell lung cancer harbouring uncommon EGFR mutations: a combined post-hoc analysis of LUX-Lung 2, LUX-Lung 3, and LUX-Lung 6. Lancet Oncol 2015;16:830-8.

(English Language Editor: C. Betlazar-Maseh) 